\title{
Microlissencephaly-micromelia syndrome
}

INSERM

\section{Source}

INSERM. (1999). Orphanet: an online rare disease and orphan drug data base.

Microlissencephaly-micromelia syndrome. ORPHA:50810

Microlissencephaly-micromelia syndrome is a syndrome of abnormal cortical

development, characterized by severe prenatal polyhydramnios, postnatal microcephaly, lissencephaly, upper limb micromelia, dysmorphic facies (coarse face, hypertrichosis, and short nose with long philtrum), intractable seizures, and early death. Hypoparathyroidism was noted in one case. 